Volume 132, Number 11, Pages 3319-3326

S 0002-9939(04)07521-5

Article electronically published on June 2, 2004

\title{
VECTOR MEASURE DUALITY AND TENSOR PRODUCT REPRESENTATIONS OF $L_{p}$-SPACES OF VECTOR MEASURES
}

\author{
E. A. SÁNCHEZ PÉREZ
}

(Communicated by N. Tomczak-Jaegermann)

The author dedicates this paper to the memory of Professor Klaus Floret.

\begin{abstract}
Let $\lambda$ be a countably additive vector measure. In this paper we use the definition of vector measure duality to establish a tensor product representation theorem for the space of $p$-integrable functions with respect to $\lambda$. In particular, we identify this space with the dual of a certain space of operators under reasonable restrictions for the vector measure $\lambda$.
\end{abstract}

\section{INTRODUCTION}

Let $(\Omega, \Sigma)$ be a measurable space, $X$ a Banach space and $X^{\prime}$ its dual space. Consider a countably additive vector measure $\lambda: \Sigma \rightarrow X$. A measurable (scalar) function $f$ is said to be integrable with respect to $\lambda$ if

1 ) it is scalarly integrable, i.e., it is integrable with respect to each scalar measure $\lambda_{x^{\prime}}(A):=\left\langle\lambda(A), x^{\prime}\right\rangle, A \in \Sigma$, and

2) for every $A \in \Sigma$ there is an element $\int_{A} f d \lambda \in X$ such that $\left\langle\int_{A} f d \lambda, x^{\prime}\right\rangle=$ $\int_{A} f d \lambda_{x^{\prime}}$ (see [1], [8] and [9]).

If $1 \leq p<\infty$, the space $L_{p}(\lambda)$ of $p$-integrable functions with respect to $\lambda$ (i.e., classes of measurable functions $f$ such that the set where they differ has zero semivariation and $|f|^{p}$ are $\lambda$-integrable) has been studied in [5] for the case $p=1$ and in [14] for every $p$. In particular, $L_{p}(\lambda)$ is a Banach function space endowed with the natural order, and its norm is given by

$$
\|f\|_{p}=\sup \left\{\left(\int_{\Omega}|f|^{p} d\left|\left\langle\lambda, x^{\prime}\right\rangle\right|\right)^{\frac{1}{p}}: x^{\prime} \in B_{X^{\prime}}\right\}, \quad f \in L_{p}(\lambda),
$$

where $\left|\left\langle\lambda, x^{\prime}\right\rangle\right|$ denotes the variation of the scalar measure $\lambda_{x^{\prime}}$. For the particular case $p=2$ these spaces have also been studied in [13], [16] and [7].

Let $p>1$ and consider the conjugate real number $p^{\prime}$, i.e., the real number that satisfies the equation $\frac{1}{p}+\frac{1}{p^{\prime}}=1$. The relation between the spaces $L_{p}(\lambda)$ and $L_{p^{\prime}}(\lambda)$ has been analyzed in [14] by means of the concept of vector measure duality. In fact, it can be proved that each function $f \in L_{p^{\prime}}(\lambda)$ can be identified with the operator $T_{f}: L_{p}(\lambda) \rightarrow X$ given by $T_{f}(g):=\int_{\Omega} f g d \lambda$ (see Proposition 8 in [14]).

Received by the editors October 23, 2002 and, in revised form, August 21, 2003.

2000 Mathematics Subject Classification. Primary 46E30; Secondary 46G10.

Key words and phrases. Vector measures, $p$-integrable functions, tensor products.

(C)2004 American Mathematical Society 
On the other hand, the spaces of 2-integrable functions with respect to a positive vector measure defined on a Banach lattice with an unconditional basis can be applied in the context of the function approximation and Fourier analysis (see [13] and [7]). The results obtained concerning the properties of these spaces suggest that the applications of the spaces $L_{p}(\lambda), 1 \leq p<\infty$, in the same context could be fruitful. The aim of this paper is to clarify their structure by means of the vector measure duality for the particular positive measures quoted above. We will restrict our attention to the case of spaces $L_{p}(\lambda)$ for $1<p<\infty$. The reason is that the case $p=1$ implies the use of the space $L_{\infty}(\lambda)$, which is out of the scope of this paper.

Thus, the second section of this paper is devoted to obtaining a tensor product representation of the spaces $L_{p^{\prime}}(\lambda)$, or (equivalently) to characterizing them as subspaces of the space of operators from $L_{p}(\lambda)$ to $X$. In Section 3 we obtain the results for positive vector measures defined on Banach lattices with an unconditional basis. In particular, this provides a new point of view for the understanding of the dual spaces of spaces of integrable functions, which have been much studied in recent years (see for example [12]).

Our notation is standard. If $X$ is a Banach space, we write $X^{\prime}$ for the dual space, and $B_{X}$ denotes the (closed) unit ball. If $x \in X, \operatorname{span}\{x\}$ is the linear span of the element $x$. The space of continuous operators from the Banach space $Y$ to the Banach space $X$ is denoted by $L(Y, X)$. If $f$ is a measurable function, we write $\operatorname{supp}\{f\}$ for its support.

\section{Operators DEFINED BY $p$-INTEGRABLE FUNCTIONS WITH RESPECT TO A VECTOR MEASURE}

Let $\lambda$ be a countably additive vector measure, and consider a real number $p>1$. The following result associates an operator $T_{f}: L_{p}(\lambda) \rightarrow X$ to each function $f \in$ $L_{p^{\prime}}(\lambda)$ by means of the integral operator. It can be deduced from [14], Section 3.

Proposition 1. Let $1<p<\infty$ and $f \in L_{p^{\prime}}(\lambda)$. Then the operator $T_{f}: L_{p}(\lambda) \rightarrow X$ defined by $T_{f}(g):=\int_{\Omega} f g d \lambda$ is well defined, and $\left\|T_{f}\right\|=\|f\|_{p^{\prime}}$.

Consider the algebraic tensor product $L_{p}(\lambda) \otimes X^{\prime}$ and the expression

$$
\sigma_{p}(z):=\inf \left\{\sum_{i=1}^{n}\left(\int_{\Omega}\left|f_{i}\right|^{p} d\left|\left\langle\lambda, x_{i}^{\prime}\right\rangle\right|\right)^{\frac{1}{p}}\left\|x_{i}^{\prime}\right\|^{\frac{1}{p^{\prime}}}: z=\sum_{i=1}^{n} f_{i} \otimes x_{i}^{\prime}\right\}
$$

that is defined for every $z \in L_{p}(\lambda) \otimes X^{\prime}$. It is clear that $\sigma_{p}$ is a seminorm on $L_{p}(\lambda) \otimes X^{\prime}$. However, it is not a norm for the general case. The following example shows this.

Example 2. Consider the sequence space $\left(l_{q},\|\cdot\|_{q}\right)$ for $1<q<\infty$. Let us denote by $e_{i}, i=1, \ldots, \infty$, the elements of the canonical basis of $l_{q}$ and $l_{q^{\prime}}$. Consider the Lebesgue measure space $([0, \infty), \Sigma, \mu)$, and take the set of probalitity measures $\mu_{i}$, $i \in N$, defined as the restrictions of $\mu$ to each interval $[i-1, i]$. It is easy to prove that the vector measure $M: \Sigma \rightarrow l_{q}$ defined by

$$
M(A):=\sum_{i=1}^{\infty} \frac{\mu_{i}(A \cap[i-1, i])}{2^{i / q}} e_{i}, \quad A \in \Sigma,
$$

is countably additive. Now, take the characteristic function $h_{i}$ of the interval $[i-1, i]$, and the element $e_{j} \in l_{q^{\prime}}, i \neq j$. The tensor $h_{i} \otimes e_{j}$ belongs to the 
tensor product $L_{p}(M) \otimes X^{\prime}$ for every $1 \leq p<\infty$, and a direct calculation shows that $\sigma_{p}\left(h_{i} \otimes e_{j}\right)=0$.

We will denote by $L_{p}(\lambda) \otimes_{\sigma_{p}} X^{\prime}$ the standard (normed) quotient space defined by the seminorm $\sigma_{p}$. We will also use the symbol $\sigma_{p}$ for the quotient norm. The completion will be denoted by $\left(L_{p}(\lambda) \hat{\otimes}_{\sigma_{p}} X^{\prime}, \sigma_{p}\right)$, and $\sigma_{p}$ will be defined for these elements by continuity in the usual way.

Definition 3. We say that an operator $T: L_{p}(\lambda) \rightarrow X$ is uniformly scalarly integral if there is a nonnegative constant $K$ such that for every $x^{\prime} \in X^{\prime}$ such that $\left\|x^{\prime}\right\|=1$,

$$
\left|\left\langle T(g), x^{\prime}\right\rangle\right| \leq K\left(\int_{\Omega}|g|^{p} d\left|\left\langle\lambda, x^{\prime}\right\rangle\right|\right)^{\frac{1}{p}}, \quad g \in L_{p}(\lambda) .
$$

We will denote by $s_{p}(T)$ the infimum of all constants $K$ and by $S_{p}(\lambda)$ the set of all uniformly scalarly integral operators.

It is clear that each uniformly scalarly integral operator $T$ is continuous and $\|T\| \leq s_{p}(T)$.

The set $S_{p}(\lambda)$ obviously becomes a linear space with the usual sum of operators and product by scalars. A direct calculation shows that $s_{p}$ is a norm. The following result establishes a representation theorem for $\left(S_{p}(\lambda), s_{p}\right)$.

Proposition 4. The spaces $\left(S_{p}(\lambda), s_{p}\right)$ and $\left(L_{p}(\lambda) \otimes_{\sigma_{p}} X^{\prime}\right)^{\prime} \cap L\left(L_{p}(\lambda), X\right)$ are isometric.

Proof. We use the so-called trace duality (see [2]). First note that for every $x^{\prime} \in X^{\prime}$ the variation of the scalar measure $\lambda_{x^{\prime}}$ satisfies the relation $\lambda_{x^{\prime}}=\lambda_{\frac{x^{\prime}}{\left\|x^{\prime}\right\|}}\left\|x^{\prime}\right\|$.

If $\phi$ is an element of $\left(L_{p}(\lambda) \otimes_{\sigma_{p}} X^{\prime}\right)^{\prime}$, suppose that it defines an operator $T_{\phi}$ : $L_{p}(\lambda) \rightarrow X$ by means of the equalities

$$
\left\langle T_{\phi}(f), x^{\prime}\right\rangle=\phi\left(f \otimes x^{\prime}\right), \quad f \in L_{p}(\lambda), x^{\prime} \in X^{\prime} .
$$

Then we can consider it as an element of the (Banach) space $\left(L_{p}(\lambda) \otimes_{\sigma_{p}} X^{\prime}\right)^{\prime} \cap$ $L\left(L_{p}(\lambda), X\right)$, and its norm is given by $\max \left\{\|\phi\|,\left\|T_{\phi}\right\|\right\}$.

Take $T \in S_{p}(\lambda)$ and let us show that it defines an element $\phi_{T}$ of the dual of the tensor product. If $z=\sum_{i=1}^{n} g_{i} \otimes x_{i}^{\prime} \in L_{p}(\lambda) \otimes_{\sigma_{p}} X^{\prime}$, then

$$
\begin{gathered}
\left|\phi_{T}(z)\right|=\left|\left\langle\phi_{T}, z\right\rangle\right| \leq \sum_{i=1}^{n}\left|\left\langle T\left(g_{i}\right), \frac{x_{i}^{\prime}}{\left\|x_{i}^{\prime}\right\|}\right\rangle\right|\left\|x_{i}^{\prime}\right\| \\
\leq s_{p}(T) \sum_{i=1}^{n}\left(\int_{\Omega}\left|g_{i}\right|^{p} d\left|\left\langle\lambda, \frac{x_{i}^{\prime}}{\left\|x_{i}^{\prime}\right\|}\right\rangle\right|\right)^{\frac{1}{p}}\left\|x_{i}^{\prime}\right\| \\
\quad=s_{p}(T) \sum_{i=1}^{n}\left(\int_{\Omega}\left|g_{i}\right|^{p} d\left|\left\langle\lambda, x_{i}^{\prime}\right\rangle\right|\right)^{\frac{1}{p}}\left\|x_{i}^{\prime}\right\|^{\frac{1}{p^{\prime}}} .
\end{gathered}
$$

Since these inequalities hold for every representation of $z$, we obtain the relation $\left|\left\langle\phi_{T}, z\right\rangle\right| \leq s_{p}(T) \sigma_{p}(z)$. Therefore, $\max \left\{\left\|\phi_{T}\right\|,\|T\|\right\} \leq s_{p}(T)$, since $\|T\| \leq s_{p}(T)$. Note that such a $T$ is compatible with the quotient defined by $\sigma_{p}$.

Now consider an element $\phi \in\left(L_{p}(\lambda) \otimes_{\sigma_{p}} X^{\prime}\right)^{\prime}$ that defines an operator $T_{\phi}$. Then, for every $g$ and $x^{\prime}$ such that $\left\|x^{\prime}\right\|=1$, we have

$$
\left|\left\langle T_{\phi}(g), x^{\prime}\right\rangle\right| \leq\|\phi\| \sigma_{p}\left(g \otimes x^{\prime}\right) \leq\|\phi\|\left(\int_{\Omega}|g|^{p} d\left|\left\langle\lambda, x^{\prime}\right\rangle\right|\right)^{\frac{1}{p}} .
$$


Therefore, $s_{p}\left(T_{\phi}\right) \leq\|\phi\| \leq \max \left\{\|\phi\|,\left\|T_{\phi}\right\|\right\}$. These inequalities show that the identification $\phi \rightarrow T_{\phi}$ defines an isometry between the subspace of elements of $\left(L_{p}(\lambda) \otimes_{\sigma_{p}} X^{\prime}\right)^{\prime}$ that define an operator of $L\left(L_{p}(\lambda), X\right)$ and $\left(S_{p}(\lambda), s_{p}\right)$.

If $X$ is a reflexive space, then the argument given in the proof above leads to the isometry

$$
\left(L_{p}(\lambda) \otimes_{\sigma_{p}} X^{\prime}\right)^{\prime}=S_{p}(\lambda) .
$$

Moreover, if $\lambda^{\prime}$ is defined on the dual space $X^{\prime}$, then we obtain

$$
\left(L_{p}\left(\lambda^{\prime}\right) \otimes_{\sigma_{p}} X\right)^{\prime}=S_{p}\left(\lambda^{\prime}\right) .
$$

Since $\left(S_{p}(\lambda), s_{p}\right)$ can be identified with the intersection of two Banach spaces we have the following result.

Corollary 5. The set $S_{p}(\lambda)$ endowed with the norm $s_{p}$ defines a Banach space of continuous operators.

Moreover, the set of operators $T_{f}$ from $L_{p}(\lambda)$ to $X$ that are defined by a function $f \in L_{p^{\prime}}(\lambda)$ can be identified isometrically with a (closed) subspace of $\left(S_{p}(\lambda), s_{p}\right)$. Recall that $\phi_{T}$ is the element of $\left(L_{p}(\lambda) \otimes_{\sigma_{p}} X^{\prime}\right)^{\prime}$ that is defined by the operator $T$ by means of the trace duality (see the proof of Proposition 4 ).

Theorem 6. The space $L_{p^{\prime}}(\lambda)$ can be isometrically identified with a (closed) subspace of $\left(L_{p}(\lambda) \otimes_{\sigma_{p}} X^{\prime}\right)^{\prime}$ by the operator $i(f):=\phi_{T_{f}}$. Moreover, for every $f \in L_{p^{\prime}}$, the following equalities hold:

$$
\|f\|_{p^{\prime}}=\left\|T_{f}\right\|=s_{p}\left(T_{f}\right)=\left\|\phi_{T_{f}}\right\| .
$$

Proof. Let $f \in L_{p^{\prime}}(\lambda)$ and consider the operator $T_{f}$ associated to $f$. Let $z=$ $\sum_{i=1}^{n} g_{i} \otimes x_{i}^{\prime} \in L_{p}(\lambda) \otimes X^{\prime}$. Then

$$
\begin{gathered}
\left|\left\langle T_{f}, z\right\rangle\right|=\left|\sum_{i=1}^{n}\left\langle\int_{\Omega} f g_{i} d \lambda, x_{i}^{\prime}\right\rangle\right| \leq \sum_{i=1}^{n} \int_{\Omega}\left|f g_{i}\right| d\left|\left\langle\lambda, x_{i}^{\prime}\right\rangle\right| \\
\leq \sum_{i=1}^{n}\left(\int_{\Omega}|f|^{p^{\prime}} d\left|\left\langle\lambda, x_{i}^{\prime}\right\rangle\right|\right)^{\frac{1}{p^{\prime}}}\left(\int_{\Omega}\left|g_{i}\right|^{p} d\left|\left\langle\lambda, x_{i}^{\prime}\right\rangle\right|\right)^{\frac{1}{p}} \\
\leq\|f\|_{p^{\prime}} \sum_{i=1}^{n}\left(\int_{\Omega}\left|g_{i}\right|^{p} d\left|\left\langle\lambda, x_{i}^{\prime}\right\rangle\right|\right)^{\frac{1}{p}}\left\|x_{i}^{\prime}\right\|^{\frac{1}{p^{\prime}}} .
\end{gathered}
$$

Therefore, $f$ defines a functional $\phi_{f} \in\left(L_{p}(\lambda) \otimes_{\sigma_{p}} X^{\prime}\right)^{\prime}$ and $\left\|\phi_{f}\right\| \leq\|f\|_{p^{\prime}}$. If $\epsilon>0$ there are $g_{0} \in B_{L_{p}(\lambda)}$ and $x_{0}^{\prime} \in X^{\prime}$ such that $\left\|x_{0}^{\prime}\right\|=1$ and

$$
\|f\|_{p^{\prime}} \leq\left\langle\int_{\Omega} f g_{0} d \lambda, x_{0}^{\prime}\right\rangle+\epsilon
$$

as a consequence of Proposition 1. Since

$$
\sigma_{p}\left(g_{0} \otimes x_{0}^{\prime}\right) \leq\left(\int_{\Omega}\left|g_{0}\right|^{p} d\left|\left\langle\lambda, x_{0}^{\prime}\right\rangle\right|\right)^{\frac{1}{p}} \leq 1
$$

we obtain the result.

However, the spaces $L_{p^{\prime}}(\lambda)$ and $\left(S_{p}(\lambda), s_{p}\right)$ do not coincide in the general case, even if $X$ is reflexive. The next example shows this. 
Example 7. Consider the Lebesgue measure space $([0,1], \Sigma, \mu)$ and let $1<p<\infty$. We use the same notation as in Example 2. Consider the (countably additive) vector measure $G: \Sigma \rightarrow l_{q}, 1 \leq q<\infty$, given by $G(A):=\sum_{i=1}^{\infty} \frac{\mu(A)}{2^{i / q}} e_{i}$. It is easy to show that $L_{p^{\prime}}(G)=L_{p^{\prime}}([0,1])$. However, it is a proper subspace of $S_{p}(G)$. For instance, if $f_{0} \in L_{p^{\prime}}(G), f_{0} \neq 0$, the operator $T_{0}(g):=\left\langle\int_{\Omega} f_{0} g d G, e_{1}\right\rangle e_{1}$ belongs to $S_{p}(G)$, but it cannot be defined as an operator $T_{f}$ for any function $f \in L_{p^{\prime}}(G)$.

This example suggests that the conditions that lead us to assure the equality between $L_{p^{\prime}}(\lambda)$ and $S_{p}(\lambda)$ (i.e., when every uniformly scalarly integral operator can be represented by a function of $\left.L_{p^{\prime}}(\lambda)\right)$ are closely related to a certain property associated to the surjectivity of the integration map $I: L_{1}(\lambda) \rightarrow X, I(f):=\int_{\Omega} f d \lambda$, $f \in L_{1}(\lambda)$ (Definition 9). This will be shown in the following section for the particular case of positive vector measures defined on spaces with an unconditional basis.

\section{The Representation theOREM FOR THE CASE OF POSITIVE VeCtor MEASURES ON SPACES WITH A BASIS}

In this section we center our attention on the case of vector measures defined on spaces with an unconditional basis. We will suppose that the unconditional constant is equal to 1 for the aim of simplicity. Our aim is to obtain representation results for the spaces $L_{p}(\lambda)$ by means of tensor products and operator spaces. As a consequence, we also obtain some information about duality for these spaces.

Throughout this section $L$ will denote a Banach lattice, and $L^{+}$its positive cone.

Definition 8. We will say that a countably additive vector measure $\lambda: \Sigma \rightarrow L$ that satisfies that $\lambda(A) \in L^{+}$for every $A \in \Sigma$ is a positive vector measure.

Spaces with an unconditional basis $\left\{e_{i}: i \in N\right\}$ can be endowed with a Banach lattice structure in a standard way (see p. 2 in [11]). We will denote also by $e_{i}$, $i \in N$ the elements of the corresponding orthogonal basis. If $L$ is such a space, its positive cone $L^{+}$is the closure of the positive linear span of the basis. Then, Definition 8 makes sense in this case.

Definition 9. Let $L$ be a Banach lattice with an unconditional basis $\left\{e_{i}: i \in N\right\}$. Let $\lambda: \Sigma \rightarrow L$ be a countably additive vector measure, and let $1 \leq p<\infty$. We will say that $\lambda$ is support complete if there exists a family of functions $\left\{g_{i}: i \in N\right\} \subset$ $L_{p}(\lambda)$ such that

1) $\int_{\Omega}\left|g_{i}\right|^{p} d \lambda \in \operatorname{span}\left\{e_{i}\right\}$, and

2) $\bigcup_{i=1}^{\infty} \operatorname{supp}\left\{g_{i}\right\}=\Omega$.

Theorem 10. Let $L$ be a reflexive space with an unconditional basis, and let $1<$ $p<\infty$. If $\lambda: \Sigma \rightarrow L$ is a positive support complete vector measure, then $L_{p^{\prime}}(\lambda)$ is isometrically isomorphic to $\left(S_{p}(\lambda), s_{p}\right)$.

Proof. First note that for every $A \in \Sigma$ and $i \in N$,

$$
\left|\left\langle\lambda, e_{i}\right\rangle\right|(A)=\left\langle\lambda, e_{i}\right\rangle(A),
$$

since $\lambda$ is positive. Let $i \in N$, and consider the set

$$
B_{i}=\left\{g \in L_{p}(\lambda): \int_{\Omega}|g|^{p} d\left\langle\lambda, e_{j}\right\rangle=0, \text { for every } j \neq i\right\} .
$$


Let $\mu$ be a Rybakov measure for $\lambda$. All the equalities and inclusions between measurable sets in the proof must be understood $\mu$-a.e.

We claim that $B_{i}$ is a projection band, and there is a set $A_{i} \in \Sigma$ such that $B_{i}=\left\{g \chi_{A_{i}} \mid g \in L_{p}(\lambda)\right\}$. Moreover, $A_{i}$ and $A_{j}$ are disjoint sets if $i \neq j$. Since $\lambda$ is positive, each element $e_{i}$ defines a positive measure $\left\langle\lambda, e_{i}\right\rangle$. Thus, if $g_{1}, g_{2} \in L_{p}(\lambda)$, $\mu_{1}, \mu_{2}$ are real numbers and $j \neq i$ we have

$$
\begin{gathered}
\left(\int_{\Omega}\left|\mu_{1} g_{1}+\mu_{2} g_{2}\right|^{p} d\left\langle\lambda, e_{j}\right\rangle\right)^{\frac{1}{p}} \\
\leq\left|\mu_{1}\right|\left(\int_{\Omega}\left|g_{1}\right|^{p} d\left\langle\lambda, e_{j}\right\rangle\right)^{\frac{1}{p}}+\left|\mu_{2}\right|\left(\int_{\Omega}\left|g_{2}\right|^{p} d\left\langle\lambda, e_{j}\right\rangle\right)^{\frac{1}{p}}=0,
\end{gathered}
$$

and then $\mu_{1} g_{1}+\mu_{2} g_{2} \in B_{i}$. Since $\|g\|_{L_{p}\left(\left\langle\lambda, e_{j}\right\rangle\right)} \leq\|g\|_{p}$ for every $g \in L_{p}(\lambda)$, we obtain that $B_{i}$ is a (closed) subspace of $L_{p}(\lambda)$. Moreover, if $|h| \leq|g|$, then $\int_{\Omega}|h|^{p} d\left\langle\lambda, e_{j}\right\rangle \leq \int_{\Omega}|g|^{p} d\left\langle\lambda, e_{j}\right\rangle$ for every $j \in N$. This means that $B_{i}$ is an ideal. But $L_{p}(\lambda)$ is an order continuous Banach lattice by Proposition 6 in [14], and then it is a projection band as a consequence of a theorem due to Ando (see Prop. 1.a.11 in 11]). The characterization of the projection bands given in Prop. 1.a.10 of [11] assures that the projection $P_{i}$ associated to the band $B_{i}$ is given by

$$
P_{i}(g)=\vee\left\{h \in B_{i}: 0 \leq h \leq g\right\}, \quad g \in L_{p}^{+}(\lambda) .
$$

The projection $P_{i}^{o}$ associated to the polar $B_{i}^{o}$ of the band $B_{i}$ is defined in the same way. If we take the weak unit $\chi_{\Omega}$ of $L_{p}(\lambda)$, it is clear that $P_{i}\left(\chi_{\Omega}\right)=\chi_{A_{i}}$ for a certain $A_{i} \in \Sigma$; this is so because $\chi_{\Omega}=P_{i}\left(\chi_{\Omega}\right)+P_{i}^{o}\left(\chi_{\Omega}\right), P_{i}\left(\chi_{\Omega}\right) \leq \chi_{\Omega}$ and $P_{i}^{o}\left(\chi_{\Omega}\right) \leq \chi_{\Omega}$.

Now we show that $B_{i} \subset B_{j}^{o}$ if $i \neq j$. First note that $B_{i} \cap B_{j}=\{0\}$ for $i \neq j$ : if $g \in B_{i} \cap B_{j}, \int_{\Omega}|g|^{p} d\left\langle\lambda, e_{k}\right\rangle=0$ for every $k \in N$, and then it is easy to see that $g=0$ since $\lambda$ is positive. Consider a function $g \in B_{i}$ and suppose that there is a function $h \in B_{j}$ such that $|g| \wedge|h|=f \neq 0$. Then $|f| \leq|g|$ and $|f| \leq|h|$, and $f \in B_{i} \cap B_{j}$ as a consequence of the fact that $B_{i}$ and $B_{j}$ are ideals. Thus, $f=0$ and we get the inclusion.

Let us prove that the bands $B_{i}$ can be written as

$$
B_{i}=\left\{g \chi_{A_{i}} \mid g \in L_{p}(\lambda)\right\} .
$$

Consider a function $g \in L_{p}(\lambda)$ and let us show that $g \chi_{A_{i}} \in B_{i}$. In the other case, there is a $j \neq i$ such that $\left\langle\int_{\Omega}|g|^{p} \chi_{A_{i}} d \lambda, e_{j}\right\rangle \neq 0$. Then there is a subset $B \subset A_{i}$ such that $\left\langle\lambda(B), e_{j}\right\rangle \neq 0$ which is a contradiction since $\lambda$ is positive. The same argument shows that it is not possible to find a function of $B_{i}$ whose support is not included in $A_{i}$. Moreover, the above paragraph shows that $\left\{A_{i}\right\}_{i=1}^{\infty}$ is a class of disjoint sets. This proves the claim. Since $\lambda$ is support complete we also have that $\bigcup_{i=1}^{\infty} A_{i}=\Omega$. This is a consequence of the fact that every $g_{i}$ in the definition of support complete satisfies $g_{i}=g_{i} \chi_{A_{i}}$ and then $\operatorname{supp}\left\{g_{i}\right\} \subset A_{i}$.

Consider an operator $T \in S_{p}(\lambda)$. Then we have for each $i \in N$ and every $g \in L_{p}(\lambda)$ the inequality

$$
\left|\left\langle T(g), e_{i}\right\rangle\right| \leq s_{p}(T)\left(\int_{\Omega}|g|^{p} d\left\langle\lambda, e_{i}\right\rangle\right)^{\frac{1}{p}} .
$$

Since $\lambda$ is support complete, there is at least a function $g_{i} \in L_{p}(\lambda)$ such that $\int_{\Omega}\left|g_{i}\right|^{p} d \lambda \in \operatorname{span}\left\{e_{i}\right\}$ for every $i \in N$, which means either $B_{i} \neq\{0\}$ or $\mu\left(A_{i}\right)=0$. In fact, the structure of the bands $B_{i}$ shows that the restriction of $L_{p}(\lambda)$ to $A_{i}$ (i.e., $\left.\left(B_{i},\|\cdot\|_{p}\right)\right)$ is isometric to $L_{p}\left(A_{i},\left.\Sigma\right|_{A_{i}},\left\langle\lambda, e_{i}\right\rangle\right)$. Thus, the expression $\tau_{i}(g):=$ 
$\left\langle T\left(g_{i} \chi_{A}\right), e_{i}\right\rangle$ defines a continuous functional on $L_{p}\left(A_{i},\left\langle\lambda, e_{i}\right\rangle\right)$ and there is a function $f_{i} \in L_{p^{\prime}}\left(A_{i},\left\langle\lambda, e_{i}\right\rangle\right)$ such that

$$
\tau_{i}\left(g \chi_{A_{i}}\right)=\int_{A_{i}} g f_{i} d\left\langle\lambda, e_{i}\right\rangle .
$$

We can also suppose that $f_{i}$ is defined in $\Omega$, and $f_{i}(w)=0$ for every $w \in \Omega-A_{i}$. Let $f$ be the measurable function given by the pointwise $\operatorname{sum} \sum_{i=1}^{\infty} f_{i}$.

The following inequalities show that $T$ can be represented by means of the function $f$. Let $C_{n}=\bigcup_{i=1}^{n} A_{i}, n \in N$. Let us define the functions $h_{n}:=\sum_{i=1}^{n} f_{i}$. It is clear that for every $n \in N$ the function $h_{n} \in L_{p^{\prime}}(\lambda)$. Moreover,

$$
\int_{\Omega}\left|f_{n}\right|^{p^{\prime}} d \lambda=\sum_{i=1}^{n} \int_{A_{i}}\left|f_{i}\right|^{p^{\prime}} d\left\langle\lambda, e_{i}\right\rangle e_{i}
$$

If $g \in L_{p}(\lambda)$ we obtain

$$
\left\|T\left(g \chi_{C_{n}}\right)\right\|=\left\|\sum_{i=1}^{n} \int_{A_{i}} g f_{i} d\left\langle\lambda, e_{i}\right\rangle e_{i}\right\|=\left\|\int_{\Omega} g h_{n} d \lambda\right\| \leq s_{p}(T)\left\|g \chi_{C_{n}}\right\|_{p} .
$$

Fix $n \in N$. For every $m>n$,

$$
\begin{gathered}
\left\|h_{m}-h_{n}\right\|_{p^{\prime}}=\sup _{g \in B_{L_{p}(\lambda)}}\left\|\int_{\Omega} g\left(h_{m}-h_{n}\right) d \lambda\right\| \\
=\left\|\sum_{i=n+1}^{m} \int_{A_{i}}\left|f_{i}\right|^{p^{\prime}} d\left\langle\lambda, e_{i}\right\rangle e_{i}\right\|^{\frac{1}{p^{\prime}}} \leq s_{p}(T) .
\end{gathered}
$$

Since $X$ is reflexive, by Th. 1.b.5 in [10] the basis $\left\{e_{i}\right\}_{i=1}^{\infty}$ is boundedly complete and $X$ is weakly sequentially complete (Th. 1.c.10 in [10]), and then there is a strong limit for every increasing bounded sequence of elements (see Th. 1.c.4 in [11]). Therefore, the element $\sum_{i=1}^{\infty} \int_{A_{i}}\left|f_{i}\right|^{p^{\prime}} d\left\langle\lambda, e_{i}\right\rangle e_{i} \in X$, and then we can prove that the function $f \in L_{p^{\prime}}(\lambda)$. Certainly, for every $A \in \Sigma$ we can define

$$
\int_{A}|f|^{p} d \lambda=\lim _{n} \int_{A}\left|h_{n}\right|^{p} d \lambda=\sum_{i=1}^{\infty} \int_{A_{i} \cap A}\left|f_{i}\right|^{p^{\prime}} d\left\langle\lambda, e_{i}\right\rangle e_{i} \in X .
$$

Thus, the other conditions of the definition of $p^{\prime}$-integrable function with respect to $\lambda$ are easily checked for $f$. Moreover, $\|f\|_{p^{\prime}} \leq s_{p}(T)$. In fact, for every function $g \in L_{p}(\lambda)$ it is clear that

$$
T(g)=\int_{\Omega} g f d \lambda=\sum_{i=1}^{\infty} \int_{A_{i}} g f_{i}\left\langle d \lambda, e_{i}\right\rangle e_{i} .
$$

Thus, $\|f\|_{p^{\prime}}=s_{p}(T)$, which completes the proof.

Corollary 11. Under the assumptions of Theorem 10,

$$
L_{p^{\prime}}(\lambda)=\left(L_{p} \otimes_{\sigma_{p}} X^{\prime}\right)^{\prime} .
$$

Thus, $L_{p}(\lambda)$ is reflexive if and only if $L_{p^{\prime}}^{\prime}(\lambda)=L_{p} \hat{\otimes}_{\sigma_{p}} X^{\prime}$. 


\section{REFERENCES}

[1] R. G. Bartle, N. Dunford and J. Schwartz, Weak compactness and vector measures, Canad. J. Math. 7 (1955), 289-305. MR 16:1123c

[2] A. Defant and K. Floret, Tensor norms and operator ideals, North-Holland Math. Studies, Amsterdam, 1993. MR 94e:46130

[3] G. P. Curbera, Operators into $L^{1}$ of a vector measure and applications to Banach lattices, Math. Ann. 293 (1992), 317-330. MR 93b:46083

[4] G. P. Curbera, When $L^{1}$ of a vector measure is an AL-space, Pacific J. Math. 162 (1994), 287-303. MR 94k:46070

[5] G. P. Curbera, Banach space properties of $L^{1}$ of a vector measure, Proc. Amer. Math. Soc. 123 (1995), 3797-3806. MR 96b:46060

[6] J. Diestel and J. J. Uhl, Vector Measures, Math. Surveys, vol. 15, Amer. Math. Soc., Providence, RI, 1977. MR 56:12216

[7] L. M. García-Raffi, D. Ginestar and E. A. Sánchez Pérez. Integration with respect to a vector measure and function approximation, Abstract and Applied Analysis 5 (2000) 207-227. MR 2002k:41046

[8] D. R. Lewis, Integration with respect to vector measures, Pacific J. Math. 33 (1970), 157-165. MR 41:3706

[9] D. R. Lewis, On integrability and summability in vector spaces, Illinois J. Math. 16 (1972), 294-307. MR 45:502

[10] J. Lindenstrauss and L. Tzafriri, Classical Banach Spaces I, Springer-Verlag, Berlin, 1977. MR 58:17766

[11] J. Lindenstrauss and L. Tzafriri, Classical Banach Spaces II, Springer-Verlag, Berlin, 1979. MR 81c:46001

[12] S. Okada, The dual space of $L^{1}(\mu)$ of a vector measure $\mu$, J. Math. Anal. Appl. 177 (1993), 583-599. MR 94m:46050

[13] S. Oltra, E. A. Sánchez Pérez and O. Valero, Spaces $L_{2}(\lambda)$ of a positive vector measure $\lambda$ and generalized Fourier coefficients, Rocky Mountain Math. J., to appear.

[14] E. A. Sánchez Pérez, Compactness arguments for spaces of p-integrable functions with respect to a vector measure and factorization of operators through Lebesgue-Bochner spaces, Illinois J. Math. 45 (2001), 907-923. MR 2003d:46055

[15] E. A. Sánchez Pérez, Spaces of integrable functions with respect to vector measures of convex range and factorization of operators from $L_{p}$-spaces, Pacific J. Math. 207 (2002), 489-495.

[16] E. A. Sánchez Pérez, Vector measure orthonormal functions and best approximation for the 4-norm, Arch. Math. 80 (2003), 177-190. MR 2004b:46034

Departamento de Matemática Aplicada, E.T.S. Ingenieros de Caminos, Canales y Puertos, Universidad Politécnica de Valencia, Camino de Vera, 46071 Valencia, Spain

E-mail address: easancpe@mat.upv.es 